\title{
Pre-End-Stage Renal Disease Care and Early Survival among Incident Dialysis Patients in the US Military Health System
}

\author{
Robert Nee $^{a, b}$ Evan Fisher ${ }^{a, b}$ Christina M. Yuan ${ }^{a, b}$ Lawrence Y. Agodoa ${ }^{c}$ \\ Kevin C. Abbott ${ }^{\mathrm{C}}$ \\ ${ }^{a}$ Nephrology Service, Walter Reed National Military Medical Center, ${ }^{b}$ Department of Medicine, Uniformed Services \\ University of the Health Sciences, and ' NIDDK, National Institutes of Health, Bethesda, MD, USA
}

\section{Keywords}

Mortality · Dialysis · Pre-end-stage renal disease nephrology care · Dialysis vascular access · United States Renal Data System $\cdot$ Military Health System

\begin{abstract}
Background: Previous reports showed an increased early mortality after chronic dialysis initiation among the endstage renal disease (ESRD) population. We hypothesized that ESRD patients in the Military Health System (MHS) would have greater access to pre-ESRD care and hence better survival rates during this early high-risk period. Methods: In this retrospective cohort study, using the US Renal Data System database, we identified $1,256,640$ patients initiated on chronic dialysis from January 2, 2004 through December 31, 2014, from which a bootstrap sample of 3,984 non-MHS incident dialysis patients were compared with 996 MHS patients. We assessed care by a nephrologist and dietitian, erythropoietin administration, and vascular access use at dialysis initiation as well as all-cause mortality as outcome variables. Results: MHS patients were significantly more likely to have had pre-ESRD nephrology care (adjusted OR [aOR] 2.9; $95 \% \mathrm{Cl} 2.3-3.7)$ and arteriovenous fistula used at dialysis initiation (aOR 2.2; 95\% Cl 1.7-2.7). Crude mortality rates peaked between the 4th and the 8th week for both cohorts but were reduced among MHS patients. The baseline adjust-
\end{abstract}

\section{KARGER}

(c) 2017 S. Karger AG, Basel

E-Mail karger@karger.com

www.karger.com/ajn ed Cox model showed significantly lower death rates among MHS vs. non-MHS patients at 6,9 , and 12 months. This survival advantage among MHS patients was attenuated after further adjustment for pre-ESRD nephrology care and dialysis vascular access. Conclusions: MHS patients had improved survival within the first 12 months compared to the general ESRD population, which may be explained in part by differences in pre-ESRD nephrology care and vascular access types.

(c) 2017 S. Karger AG, Basel

\section{Introduction}

Overall mortality rates among end-stage renal disease (ESRD) patients in the United States have declined over the last 2 decades, with steeper declines in more recent years [1]. However, survival among dialysis patients remains poor, with an adjusted mortality rate of 169 per 1,000 patient-years (PY) in 2013 [1]. Mortality risk is especially high within the first year after the initiation of maintenance dialysis [2], with only minimal improvements over the years [3]. Data from the United States Renal Data System (USRDS) showed that among incident hemodialysis (HD) patients in 2012, the adjusted mortality rate peaked at 400 per $1,000 \mathrm{PY}$ in month 2 , and decreased thereafter to 200 per 1,000 PY in month 12 [1]. 
Identifying modifiable factors that are associated with the heightened risk of death during this early dialysis period is therefore paramount to include the timing and quality of care before initiating maintenance dialysis [4]. Optimal pre-ESRD care involves early interventions to delay the progression of chronic kidney disease, management of comorbid conditions, malnutrition and metabolic complications, timely placement of vascular access, and timely initiation of dialysis [5].

Multiple studies have supported the importance of early nephrology referral and pre-ESRD care in reducing mortality after dialysis initiation, especially during the first year on dialysis [6-16]. One of the primary focuses of pre-ESRD care is the creation of an arteriovenous fistula (AVF), as recommended by the Kidney Disease Improving Global Outcomes to be the vascular access of choice for patients needing maintenance HD [17] due to its longevity and lower rates of infection and intervention. Many studies demonstrate that HD using an AVF is associated with improved survival and fewer hospitalizations compared with the complication-prone dialysis catheters [18-25].

The Military Health System (MHS) provides a unique opportunity to assess the impact of pre-ESRD care and vascular access on early survival among incident dialysis patients. The MHS is a global, comprehensive, integrated medical network within the US Department of Defense (DoD) that serves a diverse beneficiary population of 9.6 million active and retired service members and their families [26, 27]. The MHS beneficiaries enjoy a comprehensive health care benefit, with access to primary and subspecialty care through both a direct and purchased network worldwide [28]. As such, we hypothesized that patients within the MHS would have greater access to pre-ESRD care and be more likely to achieve pre-ESRD quality indicators, with associated improved early survival after chronic dialysis initiation.

\section{Materials and Methods}

This retrospective cohort study used the USRDS, which incorporates baseline and follow-up demographic and clinical data on all patients accessing the Medicare ESRD program in the United States. We identified a total of $1,257,636$ patients initiated on either HD or peritoneal dialysis from January 2, 2004 through December 31, 2014, of whom 996 (0.08\%) were MHS-incident dialysis patients. Given the proportionally small MHS sample size, we conducted a bootstrap sampling (random sample with replacement) of the non-MHS group $(n=1,256,640)$ resulting in a substantially smaller cohort $(n=3,984)$ as a comparison group. Thus, the final study cohort consisted of 3,984 non-MHS and 996 MHS patients (ratio 4:1). We assessed care by a nephrologist and kidney dietitian, erythropoietin administration, and the type of vascular access used at dialysis initiation based on the Centers for Medicare and Medicaid Services (CMS) Form 2728. The primary outcomes were the individual pre-ESRD care components and all-cause mortality during the first 12 months of dialysis initiation. This study was approved as exempted from review by the Walter Reed National Military Medical Center Institutional Review Board.

\section{Patients and Sources}

The demographics of the dialysis population in this study has been described in the USRDS Annual Data Reports for the years studied [29]. Variables included in the USRDS standard analysis files (SAFs), as well as data collection methods and validation studies, are listed on the USRDS website (www.usrds.org). The files SAF.PATIENTS were used as the primary dataset and SAF. MEDEVID for additional information coded in the CMS Form 2728. We linked these datasets with a USRDS crosswalk file that contains unique Medicare facility identification numbers and corresponding names of the dialysis facilities, which were used to define MHS beneficiaries (active-duty service members, retirees, or dependents). These patients ( $n=996)$ were initiated at military dialysis facilities across the United States from 3 branches of the Armed Forces (Army, Navy, Air Force) and were separate from patients under the care of the Department of Veterans Affairs. Our study cohort does not include MHS patients with ESRD who were initiated on dialysis at civilian dialysis facilities. The characteristics of these particular patients could not be evaluated, having been categorized as "other" under the question of current medical coverage on CMS Form 2728. Information on the TRICARE Health Plan and patient population served by the MHS, as well as features of this federal health care system are described on the MHS website (http://health.mil/I-Am-A/Media).

\section{Predictor Variables}

Covariates in our analysis included age at initiation of dialysis, year at first ESRD service, gender, race, Hispanic ethnicity, dialysis modality, pre-ESRD nephrology care, vascular access used at start of dialysis, primary cause of ESRD, estimated glomerular filtration rate (eGFR) at dialysis initiation (chronic kidney disease Epidemiology Collaboration equation), diabetes mellitus, hypertension, other comorbid conditions from the CMS Form 2728 (Table 1), tobacco use, body mass index (BMI), serum albumin, hemoglobin, employment status, and Medicare and Medicaid insurance.

\section{Statistical Analyses}

Analyses were performed using Stata 13 SE (StataCorp, College Station, TX, USA). Univariate analyses were performed with chisquare testing for categorical variables (Fisher's exact test used for violations of Cochran's assumptions) and Student $t$ test for continuous variables (Mann-Whitney test used for non-normally distributed variables). $p<0.05$ was considered statistically significant for univariate comparisons. Variables with $p<0.10$ in unadjusted analysis were introduced into multivariate analysis as covariates because of the possibility of negative confounding. Our model development used the forced entry method in which all independent variables were entered into the model at the same time without any specific sequence in which variables were entered. We used this method of regression modeling for the logistic models and the baseline Cox model (model 1). For other Cox models (models 2, 3, and 4), hierarchical regression was conducted in which the vari- 
Table 1. Baseline demographic and clinical characteristics of incident dialysis patients in the US 2004-2014, MHS vs. non-MHS (bootstrap) cohort

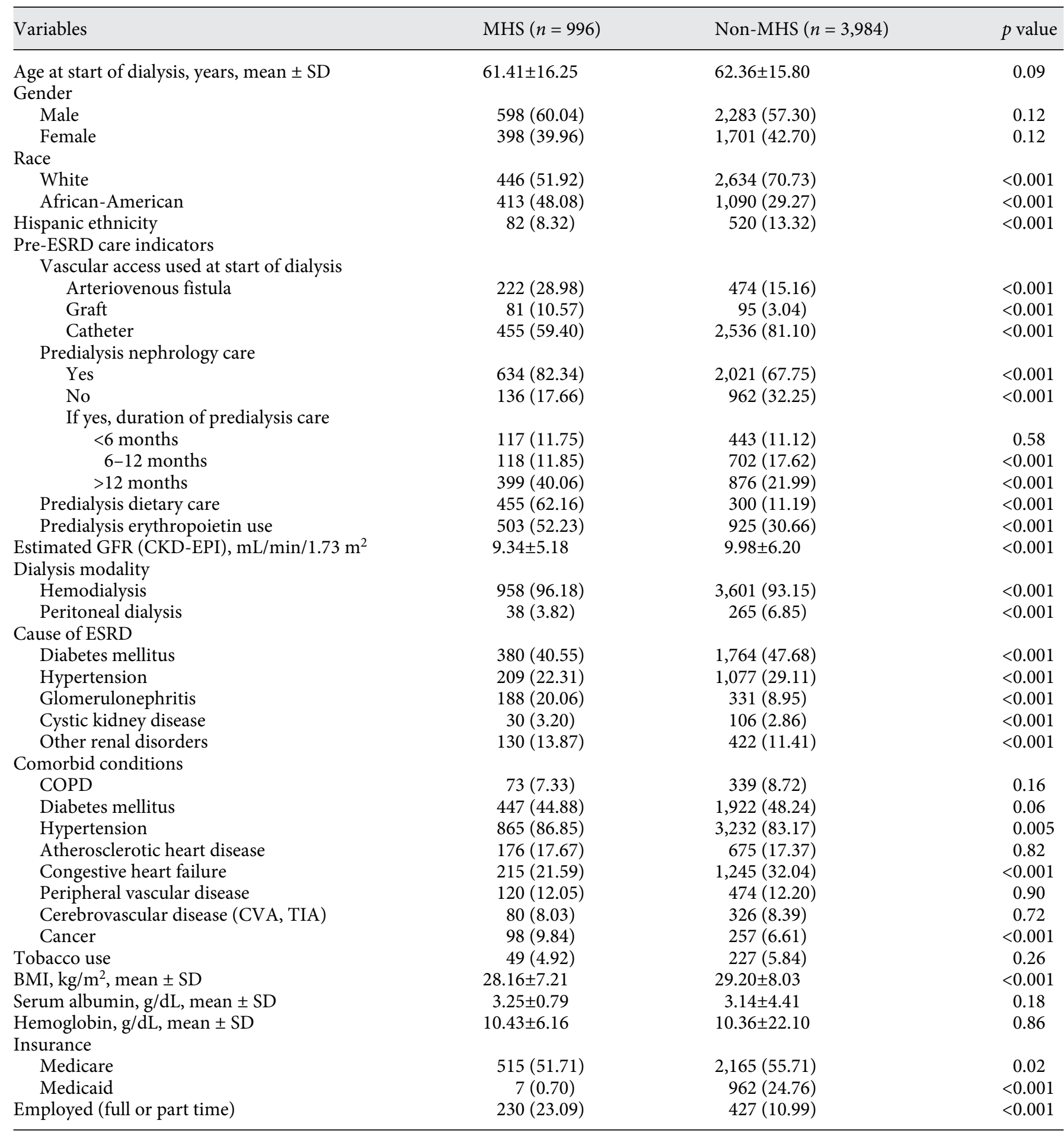

BMI, body mass index; MHS, Military Health System; ESRD, end-stage renal disease; COPD, chronic obstructive pulmonary disease; CVA, cerebrovascular disease; TIA, transient ischemic attack; GFR, glomerular filtration rate.

Data are $n(\%)$ or mean \pm SD.

Univariate analyses were performed with chi-square testing for categorical variables (Fisher's exact test used for violations of Cochran's assumptions) and Student $t$ test for continuous variables (Mann-Whitney test used for non-normally distributed variables). 
Table 2. Multivariable logistic regression models with pre-ESRD care indicators as the outcome variables

\begin{tabular}{llcc}
\hline Pre-ESRD care indicators & $\begin{array}{l}\text { Adjusted ORs } \\
\text { (MHS vs. non-MHS) }\end{array}$ & $95 \%$ CI & $p$ value \\
\hline Pre-ESRD nephrology care & 2.90 & $2.26-3.74$ & $<0.001$ \\
$\quad<6$ months & 1.16 & $0.89-1.51$ & 0.27 \\
$\quad$ 6-12 months & 0.66 & $0.51-0.84$ & 0.001 \\
$\quad$ 12 months & 2.85 & $2.36-3.44$ & $<0.001$ \\
Vascular access used at start of dialysis & 2.16 & $1.72-2.71$ & $<0.001$ \\
$\quad$ Arteriovenous fistula & 4.50 & $3.07-6.60$ & $<0.001$ \\
$\quad$ Graft & 0.33 & $0.27-0.41$ & $<0.001$ \\
$\quad$ Catheter & 16.69 & $12.97-21.47$ & $<0.001$ \\
Pre-ESRD dietary care & 2.40 & $2.00-2.90$ & $<0.001$ \\
Pre-ESRD erythropoietin use &
\end{tabular}

MHS, Military Health System; ESRD, end-stage renal disease.

$¥$ Covariables in the model included age at initiation of dialysis, year at first ESRD service, gender, dialysis modality, race, Hispanic ethnicity, primary cause of ESRD, diabetes mellitus, hypertension, chronic obstructive pulmonary disease, tobacco use, atherosclerotic heart disease, cerebrovascular disease, congestive heart failure, peripheral vascular disease, cancer, body mass index, serum albumin, hemoglobin, individual employment status, Medicare, Medicaid.

ables of interest (e.g., pre-ESRD nephrology care, vascular access) were entered one at a time, as predetermined by the investigators.

We conducted logistic regression analyses to evaluate factors independently associated with each of the pre-ESRD care components: care by a nephrologist, kidney dietitian, erythropoietin administration, and vascular access used at dialysis initiation. The logistic model correctly predicted $82.3 \%$ of the outcome variable (an observation is identified as positive if its predicted probability is $>50 \%$ ).

We also conducted Cox regression analyses to model factors associated with all-cause mortality, starting with the baseline model, which incorporated variables of demographics, dialysis modality, primary cause of ESRD, eGFR at dialysis initiation, comorbid conditions, tobacco use, BMI, and serum albumin (model 1). Model 2 was the baseline model plus the variable pre-ESRD nephrology care. Model 3 was the baseline model plus the variable vascular access types. Model 4 was fully adjusted, incorporating all the variables in the baseline model plus pre-ESRD nephrology care and vascular access types. Both graphical method and Schoenfeld residuals [30] (global test Prob > chi-square 0.63) were used to test the proportional hazards assumption.

\section{Results}

Out of a total of 1,257,636 incident dialysis patients from 2004 to 2014, we identified 996 MHS patients and a bootstrap sample of 3,984 non-MHS patients. Table 1 shows demographic and unadjusted characteristics of the study cohort. Compared to the general ESRD population, MHS patients were more likely to be African-American, employed and more likely to have glomerulonephritis as the primary cause of ESRD and cancer as a comorbid condition. On the other hand, MHS patients were less likely to have diabetes or hypertension as the primary cause of ESRD and less likely to have congestive heart failure. There were no significant differences in age, gender, and various comorbid conditions (chronic obstructive pulmonary disease, diabetes, atherosclerotic heart disease, peripheral vascular disease, cerebrovascular disease) between the 2 groups.

The proportions of MHS and non-MHS patients who were under the care of a nephrologist were 82.3 and $67.8 \%$, respectively. $62.2 \%$ of MHS patients were under the care of a kidney dietitian vs. $11.2 \%$ of non-MHS patients. 52.2\% of MHS patients received erythropoietin pre-ESRD vs. $30.7 \%$ of non-MHS patients. $29.0 \%$ of MHS patients used an AVF on dialysis initiation vs. $15.2 \%$ of non-MHS patients. On the other hand, MHS patients were less likely to initiate dialysis with a catheter compared to non-MHS patients (59.4 vs. $81.1 \%$ ).

In fully adjusted logistic regression models (Table 2), MHS patients were significantly more likely to have had pre-ESRD nephrology care (adjusted OR [aOR] 2.90); pre-ESRD dietary care (aOR 16.69); pre-ESRD erythropoietin use (aOR 2.40); and AVF on dialysis initiation (aOR 2.16). On the other hand, MHS patients were significantly less likely to initiate dialysis with a catheter compared to non-MHS patients (aOR 0.33). These findings persisted despite accounting for age, race, Hispanic ethnicity, dialysis modality, primary cause of ESRD, comorbid conditions, BMI, serum albumin, hemoglobin, insurance, and employment status. 
Fig. 1. Annualized unadjusted mortality rates of incident MHS vs. non-MHS patients during the first year following chronic dialysis initiation. MHS, Military Health System; PY, patient-years.

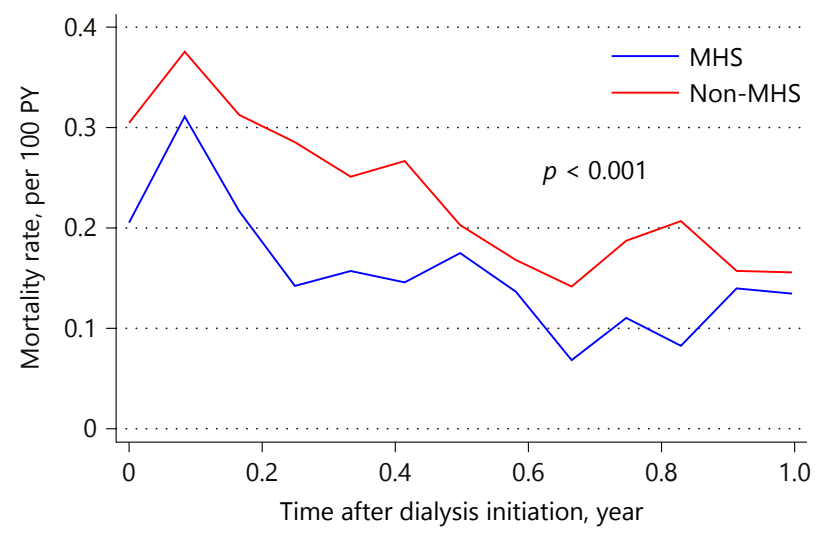

Table 3. Multivariable Cox regression models: mortality risk of MHS vs. non-MHS patients during the first 12 months after initiating chronic dialysis

\begin{tabular}{|c|c|c|c|c|c|c|c|c|}
\hline \multirow[t]{3}{*}{ Model } & \multicolumn{8}{|c|}{ AHRs (MHS vs. non-MHS) } \\
\hline & \multicolumn{2}{|l|}{3 months } & \multicolumn{2}{|l|}{6 months } & \multicolumn{2}{|l|}{9 months } & \multicolumn{2}{|l|}{12 months } \\
\hline & $\operatorname{AHR}(95 \% \mathrm{CI})$ & $p$ value & AHR (95\% CI) & $p$ value & $\operatorname{AHR}(95 \% \mathrm{CI})$ & $p$ value & $\operatorname{AHR}(95 \% \mathrm{CI})$ & $p$ value \\
\hline $1^{¥}$ (baseline) & $0.78(0.57-1.07)$ & 0.12 & $0.64(0.50-0.82)$ & $<0.001$ & $0.68(0.55-0.85)$ & 0.001 & $0.66(0.54-0.80)$ & $<0.001$ \\
\hline 2 (model $1+$ pre-ESRD nephrology care) & $0.82(0.56-1.20)$ & 0.31 & $0.70(0.52-0.95)$ & 0.024 & $0.76(0.58-0.98)$ & 0.041 & $0.72(0.56-0.91)$ & 0.006 \\
\hline 3 (model $1+$ vascular access types) & $0.84(0.57-1.22)$ & 0.36 & $0.68(0.50-0.92)$ & 0.011 & $0.75(0.58-0.96)$ & 0.028 & $0.72(0.57-0.90)$ & 0.006 \\
\hline $\begin{array}{l}4 \text { (model } 1+\text { pre-ESRD nephrology care + } \\
\text { vascular access types) }\end{array}$ & $0.93(0.63-1.38)$ & 0.73 & $0.79(0.58-1.08)$ & 0.14 & $0.84(0.65-1.10)$ & 0.21 & $0.80(0.63-1.01)$ & 0.06 \\
\hline
\end{tabular}

MHS, Military Health System; ESRD, end-stage renal disease.

$¥$ Model 1: covariates included age at dialysis initiation, gender, race, Hispanic ethnicity, year of dialysis, dialysis modality, primary cause of ESRD, estimated glomerular filtration rate at dialysis initiation, diabetes, hypertension, chronic obstructive pulmonary disease, atherosclerotic heart disease, congestive heart failure, peripheral vascular disease, cerebrovascular disease, cancer, tobacco use, body mass index, serum albumin.

Unadjusted analyses showed that MHS patients had a significantly lower mortality rate compared to non-MHS patients during the first 12 months of dialysis initiation (16 vs. 24 per 100 patient-years [PY], respectively; $p<$ 0.001 ; Fig. 1). Crude mortality rates peaked from the 4 th to the 8th week for both groups but were reduced among MHS patients ( 31 vs. 38 per $100 \mathrm{PY}, p<0.001$ ). As shown in Table 3, the baseline adjusted Cox model (model 1) showed significantly lower death rates among MHS vs. non-MHS patients at 6, 9, and 12 months. At 3 months, the adjusted hazard ratio (AHR) for death was also lower among MHS vs. non-MHS patients; however, it was not statistically significant (AHR 0.78, 95\% CI 0.57-1.07), possibly due to the low number of events (i.e., deaths) early after initiation on dialysis as compared to latter part of the year. For instance, at 3 months, 17 (1.7\%) of MHS patients had died vs. 111 (2.8\%) of non-MHS patients. At 12 months, (146) $14.7 \%$ of MHS patients had died vs. 858 $(21.5 \%)$ of non-MHS patients.
The survival advantage among the MHS vs. non-MHS patients was attenuated after adjusting for either preESRD nephrology care or vascular access types (models 2,3 ) but remained statistically significant. Pre-ESRD nephrology care and vascular access types each had a similar impact on mortality risk. The survival advantage among the MHS cohort was further attenuated after adjustment for both pre-ESRD nephrology care and vascular access types, with AHRs becoming nonsignificant (model 4).

\section{Discussion}

In a 10-year retrospective cohort study using USRDS data, we found that compared to the broader incident dialysis population, MHS patients were significantly more likely to receive pre-ESRD care, including care from a nephrologist and kidney dietician, erythropoietin administration, and AVF use at dialysis initiation. These findings 
persisted despite adjustment for age, race/ethnicity, primary cause of ESRD, employment status, insurance status, comorbidities, and other clinical factors. Specifically, MHS patients were almost 3 times as likely to receive predialysis nephrology care than non-MHS patients. When stratified by duration of care, MHS patients were significantly less likely to receive predialysis nephrology care for 6-12 months (aOR 0.66) but more likely to receive predialysis nephrology care for greater than 12 months (aOR 2.85). MHS patients were 16 times as likely to receive predialysis dietary care and about 2.5 times as likely to receive erythropoietin prior to dialysis initiation than nonMHS patients. Further, MHS patients were more likely to initiate dialysis with an AVF or graft and less likely with a catheter compared with non-MHS patients. Our results validate and expand the findings of Hurst et al. [31] who reported that patients in the Veterans Affairs and MHS systems were significantly more likely to initiate HD with an AVF than patients with other health insurance types.

The MHS' patient population, delivery systems, and quality of care parallel those found in private sector health systems in the United States $[27,28]$. This integrated and "closed" system allows its beneficiaries to be followed longitudinally, regardless of location [27]. An important basis of integration and coordination of care in the MHS is a well-developed electronic health record system that allows provider access to patients' clinical information worldwide. MHS beneficiaries enjoy a generous insurance benefit, including free or low-cost inpatient and outpatient services and prescription drugs [32]. Thus, unlike Medicare, health coverage in the MHS takes effect well before the onset of ESRD, thus facilitating predialysis care in those who are not yet eligible for Medicare due to age or disability criteria. Our findings illustrate the potential benefit of earlier access to nephrology care to optimize the patients' clinical status prior to initiation of chronic dialysis.

We found that MHS patients had a significantly lower crude mortality rate compared to non-MHS patients during the initial 12 months of dialysis initiation. Mortality rates were at the highest from the 4th to the 8th week for both cohorts but significantly reduced among MHS patients ( 31 vs. 38 per $100 \mathrm{PY}$ ). In a USRDS study of 498,566 adults initiating chronic dialysis from 2005 to 2009, Foley et al. [33] reported a peak in mortality rates (37 per 100 PY) during week 6 . Our findings are consistent with an increasing body of literature that demonstrates a heightened mortality risk within the first few months after initiation of dialysis in the United States [1,2,34] and internationally [35].

Potential factors underlying the mortality trend among incident dialysis patients include (1) early withdrawal from dialysis contributing to the elevated early mortality rates [35]; (2) ascertainment bias due to a high-risk population of patients who die soon after starting dialysis or lack Medicare coverage and are not captured in the CMS registration forms, thus underestimating the mortality rates during the initial few weeks of dialysis [2,33]; and (3) pre-ESRD nephrology care and timely placement of a permanent arteriovenous access [35]. Our baseline Cox regression model, adjusted for demographic and clinical factors, showed significantly lower risk of death among MHS vs. non-MHS patients at 6, 9, and 12 months after dialysis initiation. At 3 months, the difference in mortality rates was not statistically significant, which could be related to the low number of events (i.e., deaths) early after dialysis initiation. The survival advantage among the MHS vs. non-MHS patients was attenuated, to a similar degree, after adjusting for either pre-ESRD nephrology care or vascular access types. The survival advantage among the MHS cohort was further attenuated and became nonsignificant after adjustment for both pre-ESRD nephrology care and vascular access types. These findings suggest that salutary factors associated with pre-ESRD nephrology care and permanent arteriovenous access may possibly contribute to lower mortality rates among MHS patients during the 12 months after dialysis initiation. Prior studies have consistently found a significant inverse association between adequacy of pre-ESRD nephrology care and early mortality among incident dialysis patients $[6,14,16,34,36,37]$. Gillespie et al. [16] found that preESRD nephrology care for $>12$ months was significantly associated with initial AVF or graft use vs. catheter (aOR 11.3). Further, Chan et al. [3] observed a $61 \%$ reduction in early death risk in those patients who initiated dialysis with an AVF, consistent with many studies demonstrating lower morbidity and mortality associated with AVF vs. catheter use $[18-25,38,39]$. In our study, the likelihood of catheter use at dialysis initiation was reduced by $67 \%$ among MHS vs. non-MHS patients, which could partly explain the improved survival in the MHS cohort, given the attenuation of its survival advantage after adjusting for vascular access types in the regression model.

However, given the high risk of selection bias in observational analyses comparing outcomes of patients with different vascular access types, 2 recent published studies have called into question the true contribution of catheters to excess mortality observed in the dialysis population. In a retrospective cohort study of 2,300 patients at 5 Canadian dialysis programs, Quinn et al. [40] reported that patients who underwent a predialysis fistula attempt had a lower mortality risk than those who did not. However, only $2.3 \%$ 
of deaths were access related; thus, the excess mortality in patients with catheters did not appear to be causally related to the catheters but may instead reflect underlying poor clinical status. In a USRDS study of 115,425 incident HD patients, Brown et al. [41] reported that the group initiating dialysis with a catheter after a failed fistula placement had significantly lower mortality rates than the group who had a catheter placed first and used upon dialysis initiation (AHR 0.66, 95\% CI 0.64-0.68). Thus, the actual mortality attributed to the catheter itself may be explained to a large degree by underlying patient factors.

Also, it is possible that unobserved patient factors in our study population contributed to the improved survival among MHS patients. MHS patients were likely healthier than their counterparts in the general community. For instance, we found that MHS patients were more likely to have glomerulonephritis, and less likely diabetes mellitus, as the primary cause of ESRD compared to non-MHS patients. MHS patients were likely to be in a better socioeconomic position as reflected by the greater proportion ofemployment and $<1 \%$ having Medicaid insurance. There was a higher proportion of African-Americans with associated survival advantage among the MHS patients compared to non-MHS patients. We adjusted for these potential confounders in our survival models. However, we could not account for unobserved factors that could affect survival to include self-motivation and compliance to therapy, family and social support, ease of access to health care, socioeconomic factors (educational level, household income) and other behavioral and social determinants of health.

Our study has other limitations to include ascertainment bias related to providers' responses on CMS Form 2728, as demonstrated by Kim et al. [42] who reported substantial disagreement between information from the form and Medicare claims on the timing of earliest preESRD nephrology care. A validation study by Longenecker et al. [43] demonstrated significant underreporting of comorbid conditions on the CMS Form 2728, which may result in lower estimates of disease burden and differential bias when comparing 2 groups. There could also be underreporting bias among patients who initiated dialysis in MHS dialysis facilities given the lack of financial incentive to complete the necessary CMS forms for registration and billing purposes. As noted above, we acknowledge the possibility of selection bias to the extent that MHS patients in our study maybe inherently healthier than their non-MHS counterparts resulting in improved survival due to unobserved, residual confounders. Lastly, we cannot make conclusions about causality, given the retrospective nature of our study.
To our knowledge, this is the first comprehensive report of a national cohort of patients dialyzed in the military health facilities. We found that MHS beneficiaries had greater access to pre-ESRD care, including care by a nephrologist and kidney dietitian, and erythropoietin administration and were more likely to initiate dialysis with an AVF. Despite a similar peak in mortality rates from the 4 th to the 8th week after initiation of dialysis, MHS patients had a significant survival advantage during the first 12 months compared with their non-MHS counterparts. Salutary factors associated with pre-ESRD nephrology care and permanent arteriovenous access may possibly contribute to lower mortality rates among MHS patients during the 12 months after dialysis initiation, although unobserved patient characteristics likely play a role as well. The patient population served by the MHS has been reported to be similar to the general population in the United States in terms of the prevalence of common clinical conditions and overall health [27]. Therefore, there is a strong potential for early survival benefit in the general ESRD population if greater access to pre-ESRD care can be implemented more broadly. Consistent with the Healthy People 2020 initiative [44], our findings from the MHS cohort support ongoing efforts in the nephrology community to improve access to high-quality and comprehensive care for patients with advanced chronic kidney disease, which includes timely placement of a permanent vascular access to minimize early catheter use.

\section{Disclosure Statement}

The authors declare no conflicts of interests.

\section{Disclaimer}

The views expressed in this paper are those of the authors and do not reflect the official policy of the Department of the Army, the Department of the Navy, the DoD, National Institutes of Health or the United States government.

\section{Support and Financial Disclosures}

No authors have any declarations to make.

\section{Acknowledgments}

Part of this manuscript was presented at the American Society of Nephrology Kidney Week 2016 Annual Meeting, Chicago, IL, USA, November 17-20, 2016. 


\section{References}

1 United States Renal Data System: 2015 USRDS Annual Data Report: Epidemiology of Kidney Disease in the United States. Bethesda, National Institutes of Health, National Institute of Diabetes and Digestive and Kidney Diseases, 2015.

2 Soucie JM, McClellan WM: Early death in dialysis patients: risk factors and impact on incidence and mortality rates. J Am Soc Nephrol 1996;7:2169-2175.

3 Chan KE, Maddux FW, Tolkoff-Rubin N, Karumanchi SA, Thadhani R, Hakim RM: Early outcomes among those initiating chronic dialysis in the United States. Clin J Am Soc Nephrol 2011;6:2642-2649.

4 Arora P, Obrador GT, Ruthazer R, Kausz AT, Meyer KB, Jenuleson CS, Pereira BJ: Prevalence, predictors, and consequences of late nephrology referral at a tertiary care center. J Am Soc Nephrol 1999;10:1281-1286.

5 Obrador GT, Pereira BJ: Early referral to the nephrologist and timely initiation of renal replacement therapy: a paradigm shift in the management of patients with chronic renal failure. Am J Kidney Dis 1998;31:398-417.

6 Hasegawa T, Bragg-Gresham JL, Yamazaki S, Fukuhara S, Akizawa T, Kleophas W, Greenwood R, Pisoni RL: Greater first-year survival on hemodialysis in facilities in which patients are provided earlier and more frequent prenephrology visits. Clin J Am Soc Nephrol 2009;4:595-602.

7 Stack AG: Impact of timing of nephrology referral and pre-ESRD care on mortality risk among new ESRD patients in the United States. Am J Kidney Dis 2003;41:310-318.

8 Innes A, Rowe PA, Burden RP, Morgan AG: Early deaths on renal replacement therapy: the need for early nephrological referral. Nephrol Dial Transplant 1992;7:467-471.

9 Jungers P, Massy ZA, Nguyen-Khoa T, Choukroun G, Robino C, Fakhouri F, Touam M, Nguyen AT, Grünfeld JP: Longer duration of predialysis nephrological care is associated with improved long-term survival of dialysis patients. Nephrol Dial Transplant 2001;16: 2357-2364.

10 Kinchen KS, Sadler J, Fink N, Brookmeyer R, Klag MJ, Levey AS, Powe NR: The timing of specialist evaluation in chronic kidney disease and mortality. Ann Intern Med 2002;137: 479-486.

11 Kessler M, Frimat L, Panescu V, Briançon S: Impact of nephrology referral on early and midterm outcomes in ESRD: EPidémiologie de l'Insuffisance REnale chronique terminale en Lorraine (EPIREL): results of a 2-year, prospective, community-based study. Am J Kidney Dis 2003;42:474-485.

12 Winkelmayer WC, Owen WF Jr, Levin R, Avorn J: A propensity analysis of late versus early nephrologist referral and mortality on dialysis. J Am Soc Nephrol 2003;14:486-492.

13 Kazmi WH, Obrador GT, Khan SS, Pereira BJ, Kausz AT: Late nephrology referral and mor- tality among patients with end-stage renal disease: a propensity score analysis. Nephrol Dial Transplant 2004;19:1808-1814.

14 Khan SS, Xue JL, Kazmi WH, Gilbertson DT, Obrador GT, Pereira BJ, Collins AJ: Does predialysis nephrology care influence patient survival after initiation of dialysis? Kidney Int 2005;67:1038-1046.

15 Schwenger V, Morath C, Hofmann A, Hoffmann O, Zeier M, Ritz E: Late referral - a major cause of poor outcome in the very elderly dialysis patient. Nephrol Dial Transplant 2006;21:962-967.

16 Gillespie BW, Morgenstern H, Hedgeman E, Tilea A, Scholz N, Shearon T, Burrows NR, Shahinian VB, Yee J, Plantinga L, Powe NR, McClellan W, Robinson B, Williams DE, Saran R: Nephrology care prior to end-stage renal disease and outcomes among new ESRD patients in the USA. Clin Kidney J 2015;8: $772-780$.

17 NKF-DOQI clinical practice guidelines for vascular access. National Kidney FoundationDialysis Outcomes Quality Initiative. Am J Kidney Dis 1997;30(4 suppl 3):S150-S191.

18 Lorenzo V, Martn M, Rufino M, Hernández D, Torres A, Ayus JC: Predialysis nephrologic care and a functioning arteriovenous fistula at entry are associated with better survival in incident hemodialysis patients: an observational cohort study. Am J Kidney Dis 2004;43: 999-1007.

19 Ishani A, Gilbertson DT, Kim D, Bradbury BD, Collins AJ: Predialysis care and dialysis outcomes in hemodialysis patients with a functioning fistula. Am J Nephrol 2014;39: 238-247.

20 Astor BC, Eustace JA, Powe NR, Klag MJ Fink NE, Coresh J; CHOICE Study: Type of vascular access and survival among incident hemodialysis patients: the Choices for Healthy Outcomes in Caring for ESRD (CHOICE) Study. J Am Soc Nephrol 2005; 16 : 1449-1455.

21 Dhingra RK, Young EW, Hulbert-Shearon TE, Leavey SF, Port FK: Type of vascular access and mortality in U.S. hemodialysis patients. Kidney Int 2001;60:1443-1451.

22 Pastan S, Soucie JM, McClellan WM: Vascular access and increased risk of death among hemodialysis patients. Kidney Int 2002;62: 620-626.

23 Pisoni RL, Arrington CJ, Albert JM, Ethier J, Kimata N, Krishnan M, Rayner HC, Saito A, Sands JJ, Saran R, Gillespie B, Wolfe RA, Port FK: Facility hemodialysis vascular access use and mortality in countries participating in DOPPS: an instrumental variable analysis. Am J Kidney Dis 2009;53:475-491.

24 Polkinghorne KR, McDonald SP, Atkins RC, Kerr PG: Vascular access and all-cause mortality: a propensity score analysis. J Am Soc Nephrol 2004;15:477-486.

25 Malas MB, Canner JK, Hicks CW, Arhuidese IJ, Zarkowsky DS, Qazi U, Schneider EB,
Black JH 3rd, Segev DL, Freischlag JA: Trends in incident hemodialysis access and mortality. JAMA Surg 2015;150:441-448.

26 Military Health System and the Defense Health Agency: Military Health System Review Final Report 2014. http://www.health. mil/Military-Health-Topics/Access-CostQuality-and-Safety/MHS-Review (accessed August 15, 2016).

27 Dorrance KA, Ramchandani S, Neil N, Fisher $\mathrm{H}$ : Leveraging the military health system as a laboratory for health care reform. Mil Med 2013;178:142-145.

28 Beauvais B, Brezinski P, Schmacker E: Healthcare quality in the department of defense. Hosp Top 2008;86:33-36.

29 United States Renal Data System: 2014 Annual Data Report. http://www.usrds.org/2014/ view/v2_01.aspx (accessed May 5, 2016).

30 Therneau T, Grambsch PM: Modeling Survival Data: Extending the Cox Model. New York, Springer Publishing Co., 2001.

31 Hurst FP, Abbott KC, Raj D, Krishnan M, Palant CE, Agodoa LY, Jindal RM: Arteriovenous fistulas among incident hemodialysis patients in Department of Defense and Veterans Affairs facilities. J Am Soc Nephrol 2010; 21:1571-1577.

32 Bagchi AD, Stewart K, McLaughlin C, Higgins $\mathrm{P}$, Croghan T: Treatment and outcomes for congestive heart failure by race/ethnicity in TRICARE. Med Care 2011;49:489-495.

33 Foley RN, Chen SC, Solid CA, Gilbertson DT, Collins AJ: Early mortality in patients starting dialysis appears to go unregistered. Kidney Int 2014;86:392-398.

34 Bradbury BD, Fissell RB, Albert JM, Anthony MS, Critchlow CW, Pisoni RL, Port FK, Gillespie BW: Predictors of early mortality among incident US hemodialysis patients in the Dialysis Outcomes and Practice Patterns Study (DOPPS). Clin J Am Soc Nephrol 2007; 2:89-99.

35 Robinson BM, Zhang J, Morgenstern $\mathrm{H}$ Bradbury BD, Ng LJ, McCullough KP, Gillespie BW, Hakim R, Rayner H, Fort J, Akizawa T, Tentori F, Pisoni RL: Worldwide, mortality risk is high soon after initiation of hemodialysis. Kidney Int 2014;85:158-165.

36 Avorn J, Bohn RL, Levy E, Levin R, Owen WF Jr, Winkelmayer WC, Glynn RJ: Nephrologist care and mortality in patients with chronic renal insufficiency. Arch Intern Med 2002;162: 2002-2006.

37 Singhal R, Hux JE, Alibhai SM, Oliver MJ: Inadequate predialysis care and mortality after initiation of renal replacement therapy. Kidney Int 2014;86:399-406.

38 Ravani P, Palmer SC, Oliver MJ, Quinn RR, MacRae JM, Tai DJ, Pannu NI, Thomas C, Hemmelgarn BR, Craig JC, Manns B, Tonelli M, Strippoli GF, James MT: Associations between hemodialysis access type and clinical outcomes: a systematic review. J Am Soc Nephrol 2013;24:465-473. 
39 Xue JL, Dahl D, Ebben JP, Collins AJ: The association of initial hemodialysis access type with mortality outcomes in elderly Medicare ESRD patients. Am J Kidney Dis 2003;42: 1013-1019.

40 Quinn RR, Oliver MJ, Devoe D, Poinen K, Kabani R, Kamar F, Mysore P, Lewin AM, Hiremath S, MacRae J, James MT, Miller L, Hemmelgarn BR, Moist LM, Garg AX, Chowdhury TT, Ravani P: The effect of predialysis fistula attempt on risk of all-cause and access-related death. J Am Soc Nephrol 2017;28:613-620.
41 Brown RS, Patibandla BK, Goldfarb-Rumyantzev AS: The survival benefit of "fistula first, catheter last" in hemodialysis is primarily due to patient factors. J Am Soc Nephrol 2017;28: 645-652.

42 Kim JP, Desai M, Chertow GM, Winkelmayer WC: Validation of reported predialysis nephrology care of older patients initiating dialysis. J Am Soc Nephrol 2012;23:1078-1085.
43 Longenecker JC, Coresh J, Klag MJ, Levey AS Martin AA, Fink NE, Powe NR: Validation of comorbid conditions on the end-stage renal disease medical evidence report: the $\mathrm{CHOICE}$ study. Choices for Healthy Outcomes in Caring for ESRD. J Am Soc Nephrol 2000;11:520529 .

44 US Department of Health and Human Services. Healthy People 2020: Chronic Kidney Disease. https://www.healthypeople.gov/2020/topicsobjectives/topic/chronic-kidney-disease/objectives (accessed September 21, 2016). 